\title{
Wohlfahrtsverbände als Profiteure des sozialen Wandels?
}

\section{Ein kritischer Blick auf die Personalentwicklung}

Die deutschen Wohlfahrtsverbände sind in den vergangenen Jahrzehnten personell stark gewachsen. Um diese kaum beachteten Giganten des Wohlfahrtsstaats etwas genauer unter die Lupe zu nehmen, analysiert dieser Beitrag ihr Personalwachstum seit der Wiedervereinigung und vergleicht es mit Entwicklungen in anderen Wirtschaftssektoren. Dabei wird auch die unzureichende Datenlage thematisiert, die differenzierte Analysen erschwert.

W ohlfahrtsverbände erfahren in Deutschland wenig wissenschaftliche, politische oder massenmediale Aufmerksamkeit. Oft werden sie nur punktuell zum Thema, sei es in den Lokalteilen der Tageszeitungen wegen ihres vielfältigen Engagements vor Ort, sei es überregional, wenn etwa die Caritas einen neuen Tarifvertrag für die Altenpflege ablehnt (Klatt 2021) oder sich in einem Bezirksverband der Arbeiterwohlfahrt ein Finanzskandal auftut. In den Sozialwissenschaften ist die Zahl der Studien über Wohlfahrtsverbände, vorsichtig formuliert, überschaubar. Bis auf

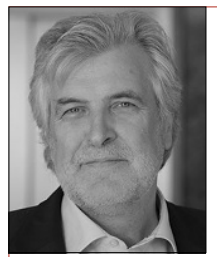

\section{Thomas Rauschenbach ${ }^{1,2}$}

'Deutsches Jugendinstitut e. V., München, Deutschland ${ }^{2}$ Technische Universität Dortmund, Dortmund, Deutschland

*1952; Studium der Erziehungswissenschaft, Psychologie und Soziologie (Dipl.-Päd., Dr. rer.soc.). Seit 1989 Professor für Theorie und Geschichte der Sozialpädagogik an der TU Dortmund, seit 2002 Direktor und Vorstandsvorsitzender des Deutschen Jugendinstituts (DJI).

rauschenbach@dji.de

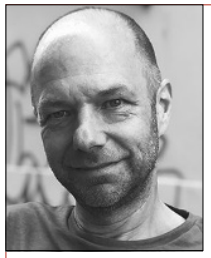

\section{Felix Berth}

Deutsches Jugendinstitut e. V., München, Deutschland *1966; Studium der Geschichte und Volkswirtschaft (M.A.), Erziehungswissenschaft (Dr. phil.). Bis 2011 Redakteur der Süddeutschen Zeitung, seit 2012 wissenschaftlicher Referent am Deutschen Jugendinstitut (DJI). berth@dji.de

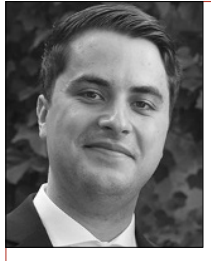

Matthias Hoffjan

Deutsches Jugendinstitut e. V., München, Deutschland *1997; Studium der Politikwissenschaft und Geschichte an der Ludwig-Maximilians-Universität München. Seit 2021 studentische Hilfskraft in der Institutsleitung des Deutschen Jugendinstituts (DJI).

hoffjan@dji.de
Zusammenfassung Der Beitrag untersucht die Personalentwicklung der Wohlfahrtsverbände in Deutschland seit 1990. Deutlich wird - trotz einer erstaunlichen Datenintransparenz ein enormes Wachstum, das in kaum einer anderen Branche des Arbeitsmarktes zu beobachten ist.

Schlüsselwörter Wohlfahrtsverbände, Personalentwicklung, Sozialstaat, Beschäftigtenstatistik

eine kurze Konjunktur der wissenschaftlichen Auseinandersetzung zu Beginn der 1990er-Jahre (Rauschenbach et al. 1995a) gibt es bis heute keine etablierten Forschungsschwerpunkte dazu.

In Anbetracht dessen zieht dieser Beitrag nach einem Vierteljahrhundert erneut eine Zwischenbilanz zur Personalentwicklung in den Wohlfahrtsverbänden (Rauschenbach und Schilling 1995b), versucht zu klären, wohin sich die Wohlfahrtsverbände im Schatten der monetären Sozialpolitik inzwischen entwickelt haben, welche gesellschaftliche Relevanz ihnen heutzutage zukommt und wie sie sich als eigener Wirtschaftszweig auf dem Arbeitsmarkt entwickelt haben. Dabei muss zugleich auf die unverändert unbefriedigende Datenlage hingewiesen werden, die differenzierte Analysen nur ansatzweise zulässt.

\section{Der Bedeutungswandel der Wohlfahrtsverbände in Deutschland}

$\mathrm{Zu}$ den Charakteristika moderner Gesellschaften gehört seit der zweiten Hälfte des 20. Jahrhunderts ihre Transformation von Industrie- zu Dienstleistungsgesellschaften sowie - darin - eine Expansion der personenbezogenen sozialen Dienste (Häußermann und Siebel 1995). Immer mehr „Dienste am Menschen“ ( $\mathrm{z}$. B. 


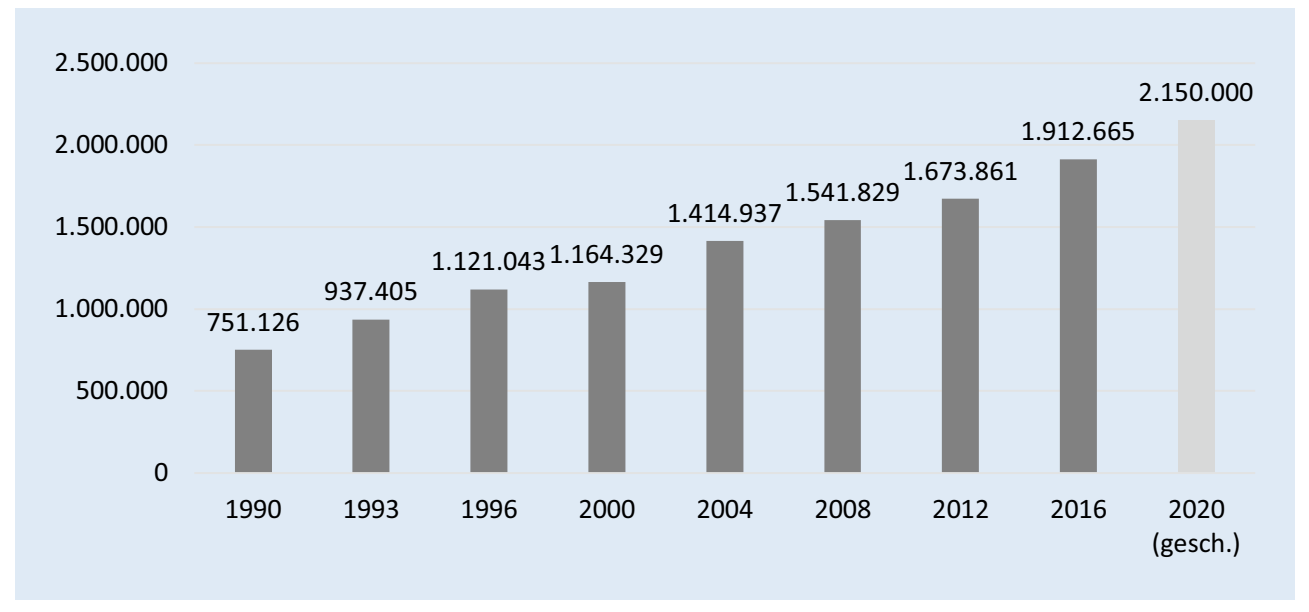

Kinderbetreuung, haushaltsnahe Dienstleistungen, Pflege behinderter, kranker und älterer Menschen), also Tätigkeiten, die zuvor ganz überwiegend in Familien und Privathaushalten erbracht wurden, sind in den letzten Jahrzehnten verstärkt zu öffentlichen Angelegenheiten geworden. In diesem Kontext wurden die Dienste und Einrichtungen gleichermaßen verrechtlicht wie monetarisiert, bürokratisiert wie professionalisiert (Sachße 1986). Oder anders formuliert: Sozialstaatliche Leistungen wurden in modernen Wohlfahrtsstaaten auf „Erwartbarkeit“ umgestellt, um Willkür, Beliebigkeit und ungleiche Hilfeleistungen zu reduzieren (Luhmann 1981). Die Leistungserbringung personenbezogener Dienste, so kann man bilanzieren, weitete sich auf diese Weise - parallel zu einer weiter bestehenden privaten Zuständigkeit - im Sinne einer wachsenden öffentlichen Verantwortung stetig aus (BMFSFJ 2013).

Dieser Trend lässt sich in vielen modernen Gesellschaften beobachten (am Beispiel der Sozialen Arbeit Züchner 2006). In Deutschland - anders als in vielen anderen Staaten - kommt den Wohlfahrtsverbänden dabei eine zentrale Stellung zu. Die sechs etablierten und in der Bundesarbeitsgemeinschaft der Freien Wohlfahrtspflege (BAGFW) zusammengeschlossenen Verbände - der katholische Caritasverband, das Diakonische Werk der Evangelischen Kirche Deutschlands, die aus der Arbeiterbewegung entstandene Arbeiterwohlfahrt, das Deutsche Rote Kreuz, der Deutsche Paritätische Wohlfahrtsverband als Dachverband für zahlreiche Einrichtungen und einzelne Fachverbände sowie die Zentralwohlfahrtsstelle der Juden in Deutschland - repräsentieren diese Entwicklung in Deutschland am deutlichsten.

Diese vom Grundsatz her mehrheitlich milieugebundenen Dienstleistungsunternehmen, die durchweg im ausgehenden 19. und frühen 20. Jahrhundert entstanden sind (Boeßenecker und Vilain 2013; Merchel 1989), konnten sich im Westen Deutschlands nach 1945 - nach ihrer Selbstauflösung oder Entmachtung im Nationalsozialismus - erneut auf breiter Ebene etablieren. In Ostdeutschland wurden sie nach der Wiedervereinigung 1990 wieder zugelassen und ersetzten das vormalige DDR-System staatlich-sozialer Einrichtungen, wobei Teile des sozialistischen Systems - etwa die auf Altenhilfe ausgerichtete „Volkssolidarität“, die inzwischen Mitglied im Paritätischen ist - in neuer Rechtsform weiterbestanden (Angerhausen et al. 1998).

\section{Die Personalexpansion der Wohlfahrtsverbände}

Die Wohlfahrtsverbände haben als Ausdruck dieser wohlfahrtspolitischen Entwicklung eine Beschäftigungsexpansion hinter sich, die auf dem deutschen Gesamtarbeitsmarkt ihresgleichen sucht. Die Zahl der Beschäftigten stieg, so die Selbstauskunft der Wohlfahrtsverbände, von rund 750.000 zum Zeitpunkt der Wiedervereinigung auf 1,9 Mio. im Jahr 2016. Und wenn man für die Zeit von 2016 bis 2021 von einem ähnlichen Wachstum wie in den Jahren davor ausgeht, dürften in den Wohlfahrtsverbänden inzwischen rund 2,15 Mio. Menschen beruflich tätig sein (BAGFW 2018; AWO 2019; Caritas 2020; Diakonie 2019; DRK 2020). ${ }^{1}$ Damit hat sich die Anzahl der Beschäftigten in den Einrichtungen und Diensten dieser Verbände innerhalb von knapp drei Jahrzehnten nahezu verdreifacht; zugleich arbeitet inzwischen jede/r fünfzehnte Erwerbstätige in Deutschland in einem Wohlfahrtsverband (Abb. 1). ${ }^{2}$

Deutlich wird neben der Dimension des Wachstums ein vergleichsweise stetiger Anstieg. Weitgehend unabhängig von ökonomischen Konjunkturen und gesellschaftlichen Diskursen expandierten die deutschen Wohlfahrtsverbände fast 30 Jahre lang. Triebfeder dieser Entwicklung waren vor allem Prozesse der Verrechtlichung der Sozialpolitik: Neue und modernisierte Gesetze im Gesundheitswesen, in der Pflege oder in der Kinder- und Jugendhilfe schufen neue Leistungssegmen- 


\section{Extrablick: Wohlfahrtsverbände in der Legitimationskrise}

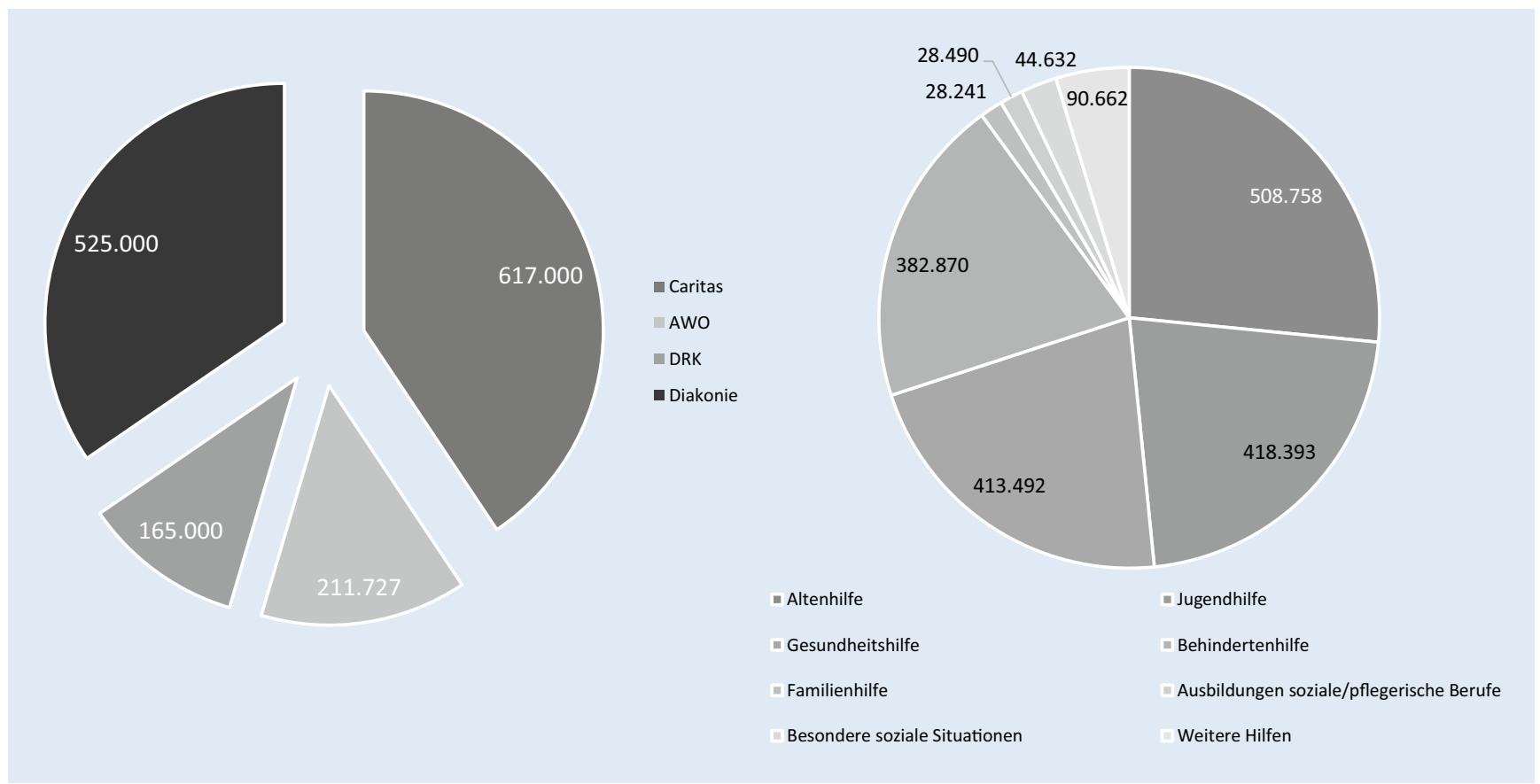

Abb. 2 Anzahl der Beschäftigten nach Einzelverbänden und nach Arbeitsbereichen in Deutschland, 2016, absolut; Quelle: BAGFW (2018); Angaben der Einzelverbände (ohne den Paritätischen und ohne die Zentralwohlfahrtsstelle der Juden); eigene Darstellung

te und rechtsverbindliche Ansprüche auf Sozialleistungen. Für Leistungsanbieter wie die Wohlfahrtsverbände entstanden Rechtssicherheit und Planbarkeit, die den Ausbau von sozialen Einrichtungen und Dienste wenig riskant erscheinen ließen. Kurz gesagt: Die Politik verabschiedete Gesetze, die Wohlfahrtsverbände schufen - vielfach federführend - die Einrichtungen, Plätze und Dienste, kümmerten sich mithin um die dafür benötigte Infrastruktur und das Personal.

Verlagert man den Blick von der Gesamtzahl der Beschäftigten auf die Größen der Einzelverbände, dann wird auch dort ein stabiler Befund sichtbar: Im Jahr 2016 war der Deutsche Caritasverband (DCV) - wie all die Jahrzehnte davor - der größte deutsche Wohlfahrtsverband, gefolgt vom Diakonischen Werk. Beide konfessionellen Verbände beschäftigten 2018 jeweils mehr als eine halbe Million Menschen (Diakonie 2019; Caritas 2020). Im Vergleich dazu haben das Rote Kreuz und die Arbeiterwohlfahrt etwa ein Drittel dieser Größe erreicht (Abb. 2). Aufschlussreich ist auch ein Blick auf die Relevanz der einzelnen „Geschäftsbereiche“ der Wohlfahrtsverbände. Sie lassen sich grob in vier ähnlich große Sektoren unterteilen: In der Altenhilfe beschäftigten die Verbände im Jahr 2016 etwa eine halbe Million Menschen, in der Kinder- und Jugendhilfe und in der Gesundheitshilfe waren es jeweils gut 400.000 Erwerbstätige. Eine ähnliche Dimension erreichte die Behindertenhilfe mit knapp 400.000 Beschäftigten; alle weiteren Hilfearten lagen deutlich unter 100.000 Beschäftigten. Insofern zeigt sich, dass „Dienste am Menschen“ das spezifische Profil der Verbände ausmachen.

\section{Die statistische Unsichtbarkeit der Wohlfahrtsverbände}

Obgleich die Wohlfahrtsverbände für den deutschen Sozialstaat wichtige personenbezogene Dienstleister sind, ist dies öffentlich kaum bekannt. Das hat auch mit ihrer statistischen Unsichtbarkeit zu tun. So ist es bei rund zwei Millionen Beschäftigten mehr als erstaunlich, wie unterbelichtet die Wohlfahrtsverbände in öffentlich zugänglichen Statistiken bleiben. Das gilt zuallererst mit Blick auf den Arbeitsmarkt, also etwa die amtlichen Statistiken zu sozialversicherungspflichtig Beschäftigten einerseits und Wirtschaftszweigen andererseits. ${ }^{3}$

Das gilt aber auch für die Statistiken der Bundesarbeitsgemeinschaft der Freien Wohlfahrtspflege (BAGFW) selbst. So findet man dort in punkto Personal einzig die Unterscheidung zwischen Vollzeit- und Teilzeitbeschäftigten, jedoch keine Informationen zu Befristungen, Geschlecht, Alter des Personals und Binnendifferenzierungen nach Verbänden. ${ }^{4}$ Obwohl ein Teil der BAGFW-Mitglieder, insbesondere die Diakonie und die Caritas, über aussagekräftige Daten aus dem eigenen Verband verfügen und diese Daten teilweise veröffentlichen, stoßen die Analysemöglichkeiten auf BAGFW-Ebene (zuletzt BAGFW 2018 mit Daten für 2016) rasch an ihre Grenzen.

Hinzu kommt eine weitere Datenlücke, der zufolge in der aktuellen BAGFW-Statistik keine Personaldaten für 


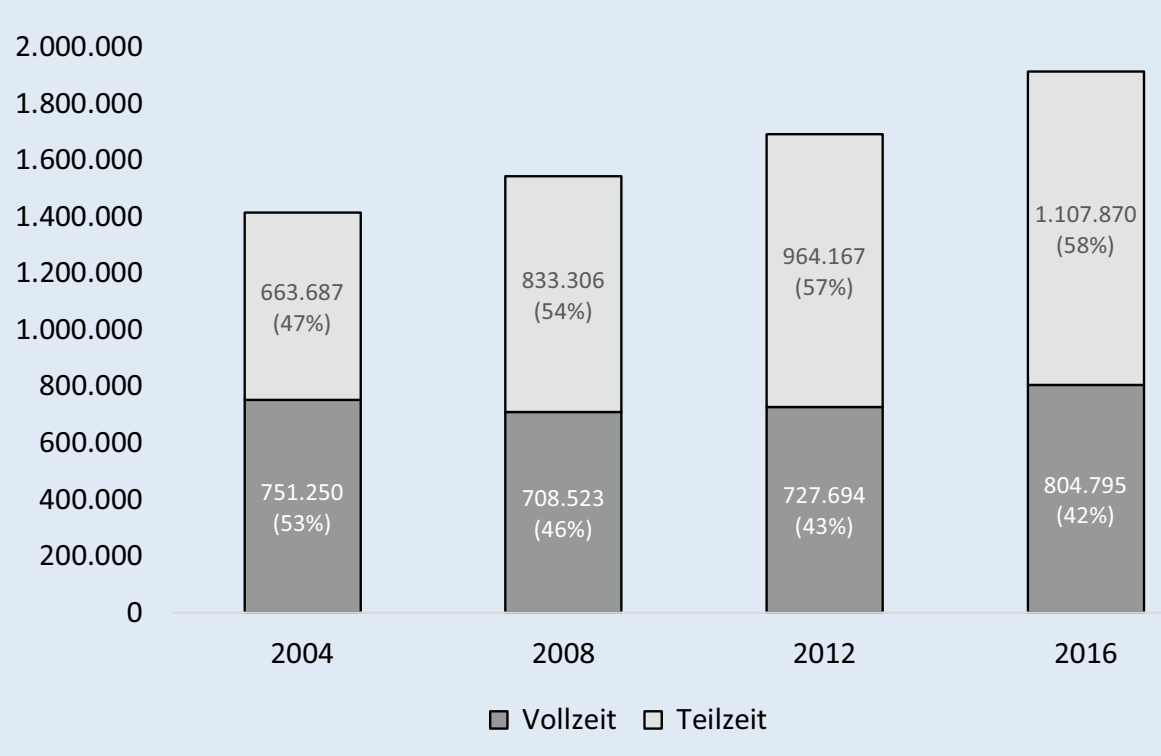

Abb. 3 Anzahl der Beschäftigten nach Einzelverbänden und nach Beschäftigungsumfang in Deutschland (2004-2016, absolut); Quelle: BAGFW (2006; 2009; 2014; 2018); eigene Darstellung den Paritätischen genannt werden. Subtrahiert man daher hilfsweise in der BAGFW-Statistik für das Jahr 2016 von der Gesamtzahl des Personals die Angaben der anderen Verbände, dann könnten zu diesem Zeitpunkt schätzungsweise bis zu 390.000 Personen in den Reihen des Paritätischen tätig gewesen sein. Allerdings: Der Paritätische selbst nennt intern eine Anzahl von 706.000 Mitarbeitenden für das Jahr 2016. ${ }^{5}$ Die gigantische Differenz der beiden Berechnungen lässt sich auf Anhieb nicht wirklich aufklären - es deutet sich jedoch das Ausmaß der Ungewissheit an, das in den Statistiken der Wohlfahrtsverbände enthalten ist, so dass man unter dem Strich nicht sicher sein kann, ob die Gesamtzahl der Beschäftigten sogar weit über zwei Millionen Personen liegt.

Weitere Datenlücken sind ebenfalls gravierend. So macht die BAGFW-Datenzusammenstellung keine Angaben zur Zahl der 450-Euro-Jobs, der geringfügig Beschäftigten oder der Honorarkräfte. Stattdessen wird ein Teil dieser Beschäftigten, die tendenziell prekärer beschäftigt sind, umstandslos der Gruppe der Teilzeitbeschäftigten zugeschlagen. Damit bleibt am Ende lediglich eine Unterscheidung zwischen Vollzeit- und Teilzeitbeschäftigten für die Jahre von 2004 bis 2016 (Abb. 3).

Obwohl diese Daten nur gut ein Jahrzehnt umfassen, wird eine markante Veränderung erkennbar: Gab es im Jahr 2004 in den deutschen Wohlfahrtsverbänden noch etwas mehr Vollzeit- als Teilzeitbeschäftigte, so hat sich dies seither grundlegend gewandelt. Im Jahr 2016 standen den etwa 800.000 Vollzeitbeschäftigten mehr als 1,1 Mio. Teilzeitkräfte gegenüber. Anders ausgedrückt: Das Personalwachstum der Verbände erfolgte fast ausschließlich bei den Teilzeitstellen, was nicht zuletzt ein
Effekt der hohen Anteile an teilzeitbeschäftigten Frauen in diesen Berufsbereichen sein dürfte. ${ }^{6}$

Insgesamt bleibt es erstaunlich, dass wir über einen derart wichtigen Teil der nationalen Wohlfahrtspolitik so wenig wissen und dass auch die politisch Verantwortlichen hier nicht mehr Transparenz einfordern und ermöglichen.

\section{Der Vergleich zu anderen Wirtschaftsbereichen}

Nichtsdestotrotz lässt sich die Bedeutung der Wohlfahrtsverbände für die Gesellschaft, die Wirtschaft und den Arbeitsmarkt wenigstens ansatzweise anhand einiger Außenvergleiche erahnen. Dabei zeigt sich - erstens - die gewachsene Relevanz der Wohlfahrtsverbände für den deutschen Arbeitsmarkt: Waren im Jahr 1990 hierzulande noch 3,3\% aller sozialversicherungspflichtig Beschäftigten in den Verbänden tätig, waren es im Jahr 2000 bereits 4,2\% und 2016 schließlich 6,1\%; dieser Wert dürfte inzwischen auf fast $6,5 \%$ gestiegen sein.

Auch im Vergleich zum öffentlichen Dienst zeigen sich - zweitens - deutliche Verschiebungen. So ist dieser sektorenübergreifend größte deutsche Arbeitgeber mit seinen etwa fünf Millionen Beschäftigten in den vergangenen drei Jahrzehnten insgesamt nicht mehr gewachsen. Wegen des Booms der Wohlfahrtsverbände haben sich die Relationen beider Bereiche jedoch deutlich verändert: Kamen im Jahr 1990 auf einen Mitarbeitenden in einem Wohlfahrtsverband noch 6,5 Beschäftigte im öffentlichen Dienst, so waren es 2016 nur mehr 2,5. Interpretieren lässt sich dies auch als ein Moment des deutschen Subsidiaritätsprinzips, wonach die öffentliche Hand nur dann selbst soziale Dienste anbietet, wenn die Freie Wohlfahrtspflege oder andere zivilgesellschaftliche Akteure dies nicht übernehmen können oder wollen. 


\section{Extrablick: Wohlfahrtsverbände in der Legitimationskrise}

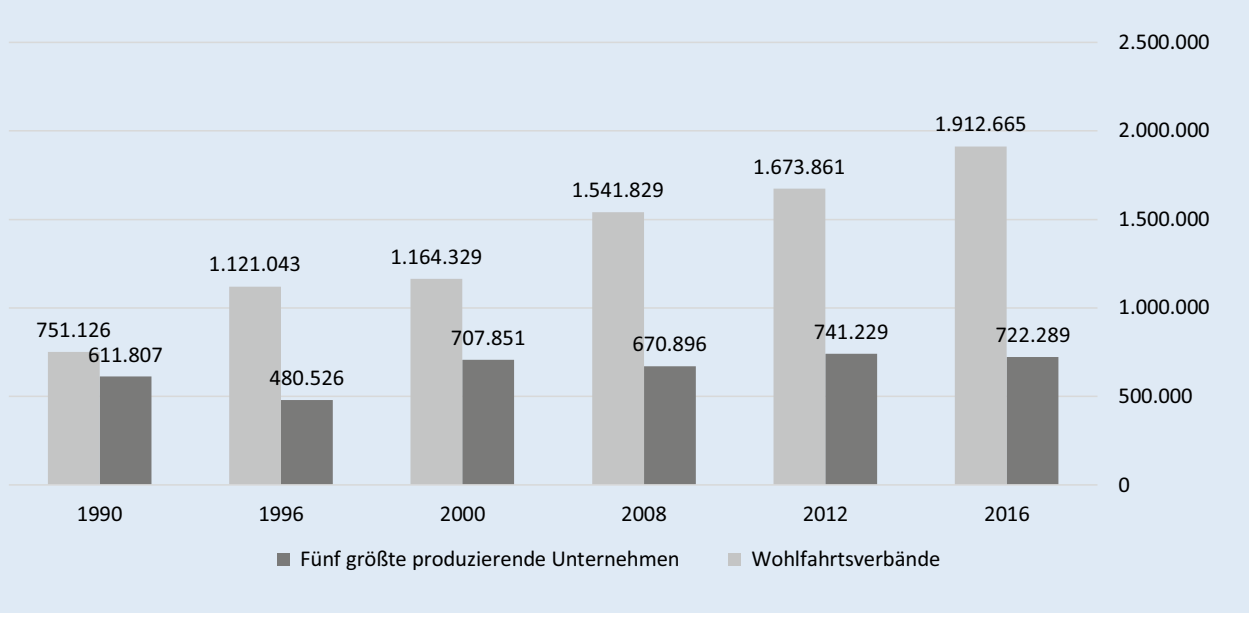

Aufschlussreich wird - drittens - ein Vergleich mit großen Wirtschaftsunternehmen. Dabei ist zu beachten, dass deutsche produzierende Unternehmen in den vergangenen Jahrzehnten neben fortschreitenden Automatisierungsprozessen große Teile ihrer Produktion in Drittstaaten mit billigeren Arbeitskräften ausgelagert und so den Globalisierungsprozess vorangetrieben haben. Am Beispiel von VW: Für das Jahr 1990 gab der Autokonzern noch 166.000 Inlandsbeschäftigte an (vgl. Rauschenbach und Schilling 1995, S. 339). Heute belaufen sich die Inlandsbeschäftigten von VW auf eine Größenordnung von 279.000. Das Gesamtwachstum des Konzerns erfolgte allerdings bis zum Jahr 2019 zu großen Teilen nicht mehr in Deutschland: Inzwischen sind knapp 56 \% des VW-Personals außerhalb der deutschen Grenzen beschäftigt (Volkswagen 2020).

Ein Vergleich der Wohlfahrtsverbände mit den aktuell größten deutschen Unternehmen des produzierenden Sektors - laut der FAZ-Datenbank sind dies die VW AG, die Robert Bosch GmbH, die Daimler AG, die Fresenius AG sowie die Continental $\mathrm{AG}^{7}$ - zeigt, wie die Entwicklungen zwischen diesen und den Wohlfahrtsverbänden auseinanderliefen. Die Zahl der Inlandsbeschäftigten dieser Industriekonzerne stieg zwischen 1990 und 2016 nur mäßig, die Wohlfahrtsverbände hingegen legten stark zu (Abb. 4). Darin verbirgt sich ein weiterer erstaunlicher Befund: Bei Caritas und Diakonie sind deutschlandweit derzeit jeweils doppelt so viele Beschäftigte tätig wie beim Autokonzern VW. Insoweit sind Wohlfahrtsverbände letzten Endes die größten privaten Arbeitgeber des deutschen Arbeitsmarkts.

\section{Eine Bilanz - und einige offene Fragen}

Das personelle Wachstum der Wohlfahrtsverbände war in den vergangenen drei Jahrzehnten - auch im Vergleich mit anderen Branchen und Sektoren - enorm. Die Zahl ihrer Beschäftigten hat sich zwischen 1990 und 2016 bzw. 2020 nahezu verdreifacht; neu entstanden sind dabei überwiegend Teilzeit-Arbeitsplätze. Die Verbände sind damit im Schatten der monetären Sozialpolitik zu stillen Riesen des deutschen Arbeitsmarktes geworden. Zugespitzt formuliert: Sie stellen eine der dynamischsten Branchen dar, die den massiven Stellenabbau in anderen Wirtschaftszweigen mit erheblichen eigenen Personalzuwächsen abgefedert und die günstige Entwicklung des deutschen Gesamtarbeitsmarktes mitgetragen haben.

Allerdings ist die Datenlage zu diesem Themenfeld insgesamt unbefriedigend und dieser gewachsenen Bedeutung nicht angemessen. So fehlt derzeit etwa das Wissen, wie viele Menschen in diesen Verbänden prekär beschäftigt sind; auch mangelt es an regionalisierten $\mathrm{Da}$ ten oder an differenzierten Befunden zu einzelnen Verbänden. Den Wohlfahrtsverbänden, so unser Resümee, scheint es nicht sonderlich angenehm zu sein, bei ihrem Personalwachstum statistisch beobachtet zu werden.

Dies setzt detaillierten Analysen deutliche Grenzen, zumal auch die amtliche Statistik dieses Thema bislang nicht in den Blick nimmt. Dennoch sollte die Bedeutung der Wohlfahrtsverbände für die Sozial- und Gesellschaftsentwicklung in Deutschland nicht unterschätzt werden. Sie zählen inzwischen zu den großen Playern des Arbeitsmarktes, auch wenn ihnen diese Position selten zugeschrieben wird und sie diese nicht für sich reklamieren.

Eingegangen. 8. Juni 2021

Angenommen. 6. August 2021

Funding. Open Access funding enabled and organized by Projekt DEAL.

Open Access. Dieser Artikel wird unter der Creative Commons Namensnennung 4.0 International Lizenz veröffentlicht, welche die Nutzung, Vervielfältigung, Bearbeitung, Verbreitung und Wieder- 
gabe in jeglichem Medium und Format erlaubt, sofern Sie den/die ursprünglichen Autor(en) und die Quelle ordnungsgemäß nennen, einen Link zur Creative Commons Lizenz beifügen und angeben, ob Änderungen vorgenommen wurden.

Die in diesem Artikel enthaltenen Bilder und sonstiges Drittmaterial unterliegen ebenfalls der genannten Creative Commons Lizenz, sofern sich aus der Abbildungslegende nichts anderes ergibt. Sofern das betreffende Material nicht unter der genannten Creative Commons Lizenz steht und die betreffende Handlung nicht nach gesetzlichen Vorschriften erlaubt ist, ist für die oben aufgeführten Weiterverwendungen des Materials die Einwilligung des jeweiligen Rechteinhabers einzuholen.

Weitere Details zur Lizenz entnehmen Sie bitte der Lizenzinformation auf http://creativecommons.org/licenses/by/4.0/deed.de.

1. Für die Jahre zwischen 2016 und heute liegen keine vollständigen Daten vor, da u. a. eine neue Gesamtstatistik der BAGFW fehlt. Doch ein Blick auf die verfügbaren Daten einzelner Verbände zeigt, dass sich der Wachstumstrend nach 2016 fortgesetzt hat: Zwischen 2016 und 2018/19 nahm das Personal der anderen Einzelverbände um etwa $13 \%$ zu, wobei es innerhalb der Verbände keine starken Abweichungen gab (DRK: $+9 \%$, AWO: $+12 \%$, Caritas: $+12 \%$, Diakonie: $+14 \%, D P W V+8 \%)$.

2. $\mathrm{Zu}$ beachten ist in diesem Zusammenhang, dass die verbandsinterne Personalstatistik lediglich zwischen Vollzeit- und Teilzeitstellen unterscheidet und in die Teilzeit auch geringfügig Beschäftigte eingerechnet werden. Insofern handelt es sich hier um „Köpfe“, nicht um Vollzeitäquivalente.

3. Abgesehen davon, dass Wohlfahrtsverbände nicht als eigener Wirtschaftszweig ausgewiesen werden, lassen sich die Beschäftigten der Woblfahrtsverbände auch nicht aus einer sogenannten "Abteilung" oder einer „Gruppe“ herausfiltern (z. B. aus der Abteilung „,86-88 Gesundheits- und Sozialwesen"), da beispielsweise die Kindertageseinrichtungen - von denen die meisten von Woblfahrtsverbänden betrieben werden - in der Abteilung „85 Erziehung und Unterricht“ eingeordnet sind. Mit anderen Worten: Wohlfahrtsverbände als Gesamtkorpus sind in der amtlichen Statistik nicht $z u$ finden.

4. Es ist erstaunlich, dass die BAGFW fast resignativ konstatiert, dass die einzelnen Verbände unterschiedliche Erhebungswege nutzen, um ibre Daten zusammenzutragen. Offenbar ist es der BAGFW bis heute nicht gelungen, ein gemeinsames Erhebungskonzept zu entwickeln und anzuwenden (so nachzulesen in den methodischen Erläuterungen zur letzten BAGFW-Statistik aus dem Jahre 2018). Es scheint kein ernsthaftes Interesse der Verbände zu geben, die eigene Entwicklung transparent aufzubereiten und öffentlich darzustellen.

5. Diese Berechnung wurde den Autoren vom Paritätischen auf Anfrage zur Verfügung gestellt und ist unseres Wissens bislang nicht öffentlich publiziert.

6. Ob sich dieser Trend in den vergangenen fünf Jahren fortgesetzt hat, lässt sich mit diesen Daten nicht abschätzen. Möglicherweise kam diese Entwicklung auch zu einem Stillstand, den man beispielsweise im Kita-Bereich beobachten konnte: Hier blieb das Verhältnis Vollzeit zu Teilzeit trotz Personalwachstums zwischen 2016 und 2020 konstant.

7. Die Frage nach den größten deutschen Industrieunternehmen in punkto Personal genau zu beantworten, ist auf mehrfache Weise schwierig. Einige große Konzerne veröffentlichen keine Personalzablen; andere weisen Mitarbeitendenzahlen nur noch global aus. Die hier als größte produzierende Unternehmen Genannten stammen aus einer FAZ-Datenbank; diese Angaben wurden durch eigene Recherchen ergänzt. Diese Liste ist allerdings eher als Annäherung an dieses Feld $z u$ verstehen, nicht als definitives Ranking.

\section{Literatur}

Angerhausen, S., et al. (1998). Überholen ohne einzuholen. Wohlfahrtsverbände in Ostdeutschland. Wiesbaden: Westdeutscher Verlag.

Arbeiterwohlfahrt (2019). Die AWO in Zahlen und Fakten (WebsiteBeitrag). https://www.awo.org/die-awo-zahlen-und-fakten. Zugegriffen: 30.9.2021.

Boeßenecker, K.-H., \& Vilain, M. (2013). Spitzenverbände der Freien Woblfahrtspflege. Eine Einführung in Organisationsstrukturen und Handlungsfelder sozialwirtschaftlicher Akteure in Deutschland. Weinheim: Beltz Juventa.

Bundesarbeitsgemeinschaft der Freien Wohlfahrtspflege (BAGFW) (2006). Gesamtstatistik 2004. Einrichtungen und Dienste der Freien Woblfahrtspflege. Berlin: BAGFW.

Bundesarbeitsgemeinschaft der Freien Wohlfahrtspflege (BAGFW) (2009). Gesamtstatistik 2008. Einrichtungen und Dienste der Freien Wohlfahrtspflege. Berlin: BAGFW.

Bundesarbeitsgemeinschaft der Freien Wohlfahrtspflege (BAGFW) (2014). Gesamtstatistik 2012. Einrichtungen und Dienste der Freien Wohlfahrtspflege. Berlin: BAGFW.

Bundesarbeitsgemeinschaft der Freien Wohlfahrtspflege (BAGFW) (2018). Gesamtstatistik 2016. Einrichtungen und Dienste der Freien Woblfahrtspflege. Berlin: BAGFW.

Bundesministerium für Familie, Senioren, Frauen und Jugend (BMFSFJ) (2013). Vierzehnter Kinder- und Jugendbericht. Bericht über die Lebenssituation junger Menschen und die Leistungen der Kinder- und Jugendhilfe in Deutschland. Berlin: BMFSFJ.

Deutscher Caritasverband (2020). Millionenfache Hilfe - Die Caritas in Zahlen (Website-Beitrag). https://www.caritas.de/diecaritas/wir-ueber-uns/ die-caritas-in-zahlen/statistik. Zugegriffen: 30.9.2021.

Deutsches Rotes Kreuz (2020). Jahrbuch 2019. https://www.drk.de/fileadmin/user_upload/PDFs/Jahrbuecher/2019_Jahrbuch/200430_DRK_Jahrbuch_2019_RGB_RZ-Doppelseiten.pdf. Zugegriffen: 30.9.2021.

Diakonie Deutschland - Evangelisches Werk für Diakonie und Entwicklung e. V. (2019). Einrichtungsstatistik 2018. https://www.diakonie.de/ statistik/092019-einrichtungsstatistik-2018. Zugegriffen: 30.9.2021.

Häußermann, H., \& Siebel, W. (1995). Dienstleistungsgesellschaften. Frankfurt a.M.: Suhrkamp.

Klatt, T. (2021). Das Veto der Caritas und seine Folgen. Deutschlandfunk. https://www.deutschlandfunk.de/pflegetarifvertrag-das-veto-der-caritas-und-seine-folgen.886.de.html?dram:article_id=493844. Zugegriffen: 30.9.2021.

Luhmann, N. (1981). Politische Theorien im Wohlfahrtsstaat. München: Olzog.

Merchel, J. (1989). Der Deutsche Paritätische Wohlfahrtsverband. Weinheim: Deutscher Studienverlag.

Rauschenbach, T., \& Schilling, M. (1995). Die Dienstleistenden. Wachstum, Wandel und wirtschaftliche Bedeutung des Personals in Wohlfahrts- und Jugendverbänden. In T. Rauschenbach, et al. (Hrsg.), Von der Wertgemeinschaft zum Dienstleistungsunternehmen. Jugend- und Wohlfahrtsverbände im Umbruch (S. 321-355). Frankfurt a.M.: Suhrkamp.

Rauschenbach, T., Sachße, C., \& Olk, T. (1995a). Von der Wertgemeinschaft zum Dienstleistungsunternehmen. Jugend-und Woblfahrtsverbände im Umbruch. Frankfurt a.M.: Suhrkamp.

Sachße, C. (1986). Mütterlichkeit als Beruf. Sozialarbeit, Sozialreform und Frauenbewegung 1871-1929. Wiesbaden: Springer VS.

Volkswagen AG (2020). Mobilität für kommende Generationen - Geschäftsbericht 2019. https://www.volkswagenag.com/presence/investorrelation/publications/annual-reports/2020/volkswagen/Y_2019_d.pdf. Zugegriffen: 30.9 .2021 .

Züchner, I. (2006). Soziale Arbeit - Aufstieg im Schatten des Woblfahrtsstaates. Weinheim: Beltz Juventa. 